\title{
Factors Affecting Success of Semi-rigid Ureterorenoscopy in Proximal Ureter Stone Treatment
}

\author{
Sezgin Okçelik ${ }^{1}$, Niyazi Ozgur Kurul ${ }^{2}$, Halil Kiziloz ${ }^{1}$, Muhammed Cihan Temel ${ }^{1}$ and Cumhur Yesildal ${ }^{3}$ \\ ${ }^{1}$ Department of Urology, Nevşehir State Hospital, Turkey \\ ${ }^{2}$ Department of Urology, Düziçi State Hospital, Turkey \\ ${ }^{3}$ Department of Urology, Sultan II, Abdulhamid Han Teaching and Research Hospital, İstanbul, Turkey
}

\begin{abstract}
Objective: To evaluate the factors affecting the success of semi-rigid ureteroscopy in proximal ureter stones.

Study Design: Descriptive study.

Place and Duration of Study: Department of Urology, Nevşehir State Hospital, Turkey between March 2017 and October 2019.

Methodology: Patients, who underwent a semi-rigid ureteroscopic lithotripsy (URSL) operation for proximal ureteral stones, were evaluated retrospectively in terms of gender, age, stone side, stone size, stone density, the type of lithotripsy, placement of the stone cone, the type of anesthesia and postoperative stone-free rates, which were recorded. The diameter of the ureter with the stones and the distances of the stones to the ureteropelvic junction (UPJ) were measured. Patients who had stone-free status after the URSL were labelled as group I. Patients whose stones were pushed back during URSL were labelled as group II.

Results: The distance of the stone to the UPJ was statistically significantly higher in group I $(p=0.006)$. The rate of using stone cone in patients in group I was statistically significantly higher than in patients in group II $(p=0.001)$. The rate of stones in the middle ureter in group I was statistically higher than group II $(p<0.001)$. The rate of using laser lithotriptor in group I was statistically higher than group II $(p=0.007)$.

Conclusion: Semi-rigid URSL is a useful technique in the proximal ureter stones. The distance of the stone to the UPJ affects the success; and using laser lithotripsy and stone cone increases the success.
\end{abstract}

Key Words: Ureteral stone, Ureterorenoscopy, Laser lithotriphsy, Pnomotic lithotripsy.

How to cite this article: Okçelik S, Kurul NO, Kiziloz H, Temel MC, Yesildal C. Factors Affecting Success of Semi-rigid Ureterorenoscopy in Proximal Ureter Stone Treatment. J Coll Physicians Surg Pak 2021; 31(01):65-69.

\section{INTRODUCTION}

Ureteral stone may cause obstructive uropathy and hydronephrosis, which may eventually make renal function deteriorate. ${ }^{1}$ The main purpose of ureteral stone treatment is to remove the stone completely with minimal morbidity. Urologists consider many factors, such as location of stone, stone size, patient's age, degree of hydro nephrosis, symptom severity, accompanying bacterial infection, status of solitary kidney, patient expectations and technical equipment, when making treatment decisions. ${ }^{2}$ Stones smaller than $5 \mathrm{~mm}$ are more suitable for spontaneous passage through the ureter. ${ }^{3}$ Proximal ureteral stones are defined as stones which are located above the iliac cross. ${ }^{4}$ Proximal ureteral stones were shown to be more difficult to pass spontaneously compared to distal ureteral stones. ${ }^{5}$

Correspondence to: Dr. Sezgin Okçelik, Department of Urology, Nevşehir State Hospital, Turkey

E-mail:drsezginokcelik@hotmail.com

Received: May 21, 2020; Revised: December 21, 2020;

Accepted: December 30, 2020

DOI: https://doi.org/10.29271/jcpsp.2021.01.65
With the advances in technology, rigid and flexible ureteroscopy (f-URS) may be used instead of shock wave lithotripsy (SWL), in the treatment of proximal ureteral stones. ${ }^{6,7}$ Pneumatic lithotripter and holmium:yttrium-aluminum-garnet (Ho:YAG) laser can be used for intracorporeal lithotripsy. ${ }^{8}$

In this study, the factors affecting the success of semi-rigid ureteroscopic lithotripsy (URSL) in proximal ureter stones were assessed. Proximal ureteral stone treatment is increasingly shifting to ureteroscopic lithotripsy. Knowing the factors that affect the success of ureteroscopic lithotripsy will be useful for the technical option.

The aim of this study was to evaluate the distance between the stone and the UPJ, the diameter of the ureter in the proximity of the stone and the effect of these parameters on the success of the surgical procedure.

\section{METHODOLOGY}

After obtaining the approval of the local Ethics Committee, assessed patients, who had received semi-rigid ureterorenoscopic lithotripsy operation for proximal ureteral at stones at Nevşehir State Hospital, Turkey between March 2017 and October 2019, were evaluated. The authors used 9 Fr RZ Mediz- 
intechnik $^{T M}$ ureteroscope, Vibrolith intracorporeal pneumatic lithotripter, ${ }^{\mathrm{TM}}$ and Quanta System Litho ${ }^{\mathrm{TM}} 35$ W Holmium:Yag laser for lithotripsy, and Boston Scientific Stone Cone ${ }^{\mathrm{TM}}$ stone retrieval coil. The patient data was evaluated retrospectively, and the age, gender, stone side, size of the stone, stone density, lithotriptor type (holmium laser / pneumatic), whether a stone cone was used, anesthesia type, and postoperative stone-free rateswere recorded. Inaddition, non-contrastcomputed tomography (NCCT) images were analysed and the diameters of the ureters in the proximity of the stones and the distance of stones to the UPJ were evaluated. The distance from UPJ to proximal of the stone on the software were measured (Figure 1). General stone-free rates and the effect of the above-mentioned parameters on stone-free rates were evaluated. Evaluation was performed three weeks after operation with ultrasonography and NCCT. Patients, whose operations were successful and whose stone was removed, were classified as successful and named as group I. Patients, whose stones were pushed back during the operation, were classified as unsuccessful and named as group II. Patients with urinary anomalies, urinary infection, multiple ureteral stones and pregnant patients were excluded from the study. The inclusion criterias were: age above 18 years, a sterile urinary system, and $<2 \mathrm{~cm}$ proximal ureteralstone.

SPSS 17.0 (Chicago, Illinois, USA) was used for analysing the data. Shapiro-Wilk test was used to determine normality. Qualitative variables were shown in numbers with percenteges, where quantitative variables in mean with standard deviation and median with inter-quartile range (IQR). Chi-square/Fischer's exact test and Mann-Whitney U-test were used to compare the groups. A p-value of less than 0.05 was considered to show a statistically significant result.

\section{RESULTS}

One hundred and fifty-three patients, who were admitted to the outpatient clinic between March 2017 and October 2019 and diagnosed with proximal ureteral stones, were evaluated. Forty-nine patients were excluded from the study because of missing information at their NCCT images. The mean age of the 104 patients was $43.8 \pm 12.77$ years. Seventy-five patients $(72.1 \%)$ were males and 29 (27.9\%) were females. Factors affecting the results of the semi-rigid URSL in proximal ureteral stone treatment are shown in Table I. Stone-free rate in patients after operation was 82 (78.8\%, group I). In $22(21.2 \%)$ patients, stones were pushed back during the operation (group II). The overall success rate of the operation was $78.8 \%$. There was no statistically significant difference between the groups in terms of stone size, stone density and proximal ureter calibre ( $p$ $=0.903,0.338,0.345$, respectively). Further, the stone was to the UPJ resulted in a significantly higher successful operation group ( $p=0.006)$. The rate of using stone cone in patients in the successful group was significantly higher than in patients in push-backgroup $(p=0.001)$.

Of the ureter stones, $57(54.8 \%)$ were on the right and $47(45.2 \%)$ on the left side. Of the patients in group I, the stone was on the right side of 43 (52.4\%) patients and on the left side of $39(47.6 \%)$ patients. In group II, the stone was on the right side of $14(63.6 \%)$ patients and on the left side of $8(36.4 \%)$ patients. There was no statistically significant difference between the patients in group I and group II in terms of stone side $(p=0.349)$.

There were $62(59.6 \%)$ stones in the upper ureter and 42 $(40.4 \%)$ stones in the middle ureter of all the patients. In group I, $40(48.8 \%)$ patients had an upper ureteral stone and 42 (51.2\%) had a middle ureteral stone. In group II, all the stones were at the upper ureter. Group I had more stones in the middle ureter than group II $(p<0.001)$.

Holmium laser lithotripsy was used in 68 (65.4\%) patients, and pneumatic lithotripsy in 36 (34.6\%) patients. In group I, 59 laser lithotripsy (72\%) and 23 pneumatic lithotripsy (28\%) treatments were performed. In group II, nine laser lithotripsy (40.9\%) and 13 pneumatic lithotripsy $(59.1 \%)$ treatments were performed. The use of laser lithotripsy increased the success of ureteroscopy in proximal ureter stones ( $p=0.007$ ). In 21 $(25.6 \%)$ patients, general anesthesia was given and in 61 (74.4\%) patients spinal anesthesia was used in group I. In five (22.7\%) patients, general anesthesia was given; and in 17 (77.3\%) patients, spinal anesthesia was used in group II. There was no statistically significant difference in anesthesia between the groups $(p=0.782)$. D-J stent was placed in $74(71.2 \%)$ patients after surgery.

\section{DISCUSSION}

Ureteral stones are important health problem throughout the world. Patients are admitted to the emergency room and urology outpatient clinics, mostly with renal colic. ${ }^{9}$ Medical expulsive therapy, SWL, and ureteroscopy are treatment options. $^{3,10}$

While the first option for proximal ureteral stones was SWL, URSL has come to the fore with the development of technology and the use of semi-rigid and flexible URS with Ho: YAG lase. ${ }^{10}$ Considering the success of semi-rigid URSL, the treatment of distal ureteral stones is more successful, but the rate of success in proximal stones increases with the developing technology (98\%; $80 \%$ respectively). ${ }^{11}$ Several studies have been done to find out the factors affecting the success of URSL for proximal ureteral stones. ${ }^{2,12}$ Many factors affect the ureteroscopy's success, like age, gender, stone size, stone density, impacted stone history, and lithotripter type (laser or pneumatic). 2,12 Unlike previous studies, the effects of these factors on stonefree rates by measuring the distance to the UPJ and the ureter diameter above the stone by examining the NCCT images were evaluated.

Sen, Sancak, Günlüsoy and Bangash reported that stone size is associated with the success rate of semi-rigid ureteroscopy in proximalureteral stone. . $^{2,6,10,12}$

There are studies that claim the opposite. ${ }^{13-15}$ In this study, the size of stones were similar in both the groups $(p=0.903)$. The presentstudy supports the reports of Chen and Cheung. ${ }^{13,14}$ 
Table I: Factors affecting the results of the semi-rigid URSL in proximal ureteral stone treatment.

\begin{tabular}{|c|c|c|c|}
\hline & Group I (n=82) & Group II (n=22) & p-value \\
\hline Stone density (HU) & $716(410.75-1028.75)$ & 837.5 (549.75-1035.25) & 0.338 \\
\hline Stone size $(\mathrm{mm})$ & $8(6-11)$ & $8(5.6-11)$ & 0.903 \\
\hline Proximal ureter caliber (mm) & $10(7-13)$ & $10(9-13.25)$ & 0.345 \\
\hline The distance of the stone to the UPJ (mm) & $45.5(28-70)$ & $27.5(20-41.5)$ & 0.006 \\
\hline Stone cone placement $(\mathrm{n})$ & $25(30.5 \%)$ & $0(0 \%)$ & 0.001 \\
\hline Right side $(\mathrm{n})$ & $43(52.4 \%)$ & $14(63.6 \%)$ & \multirow{2}{*}{0.349} \\
\hline Left side $(n)$ & $39(47.6 \%))$ & $8(36.4 \%)$ & \\
\hline Upper ureter $(n)$ & $40(48.8 \%)$ & $22(100 \%)$ & \multirow{2}{*}{$<0.001$} \\
\hline Middle ureter(n) & $42(51.2 \%)$ & $0(0 \%)$ & \\
\hline Laser lithotripsy $(n)$ & $59(72 \%)$ & $9(40.9 \%)$ & \multirow{2}{*}{0.007} \\
\hline Pneumatic lithotripsy (n) & $23(28 \%)$ & $13(59.1 \%)$ & \\
\hline General anesthesia (n) & $21(25.6 \%)$ & $5(22.7 \%)$ & \multirow{2}{*}{0.782} \\
\hline Spinal anesthesia (n) & $61(74.4 \%)$ & $17(77.3 \%)$ & \\
\hline
\end{tabular}

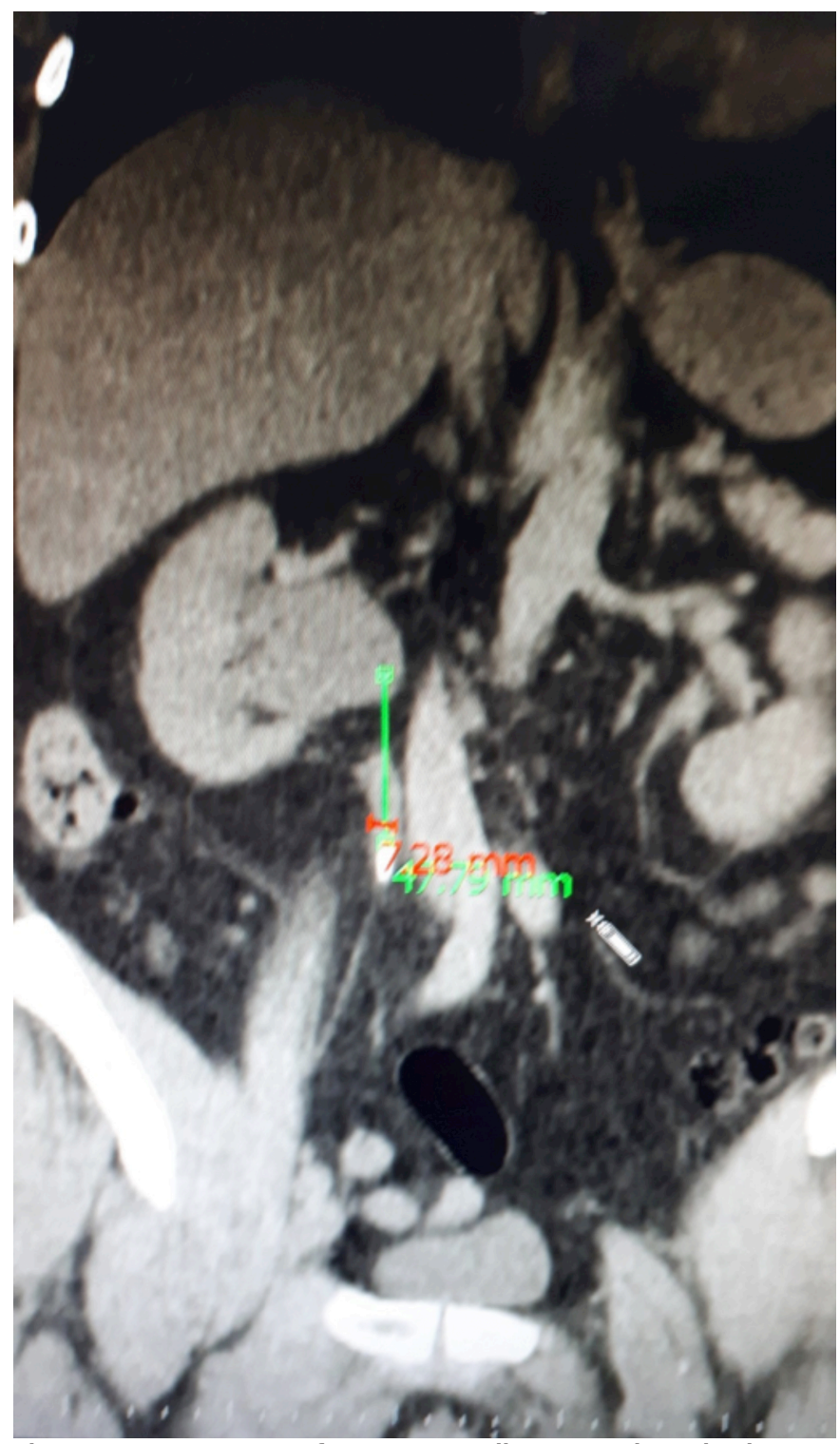

Figure 1: Measurement of stone to UPJ distance and proximal ureter width.

The articles evaluating the effects of stone density measured with the Hounsfield unit are also available in the literature. ${ }^{16}$
Considering that the lithotripsy process will be more difficult in harder stones, failure rates are thought to be higher. In this study, no difference was observed between the two groups in terms of stone density. The results of the present study are similar to those of Sen et al. ${ }^{2}$

It was observed that stone side did not affect the success of semi-rigid ureteroscopy. There was no difference in the side of stones, as shown in the Kaygisız and collegues' study, supports the work of this study. ${ }^{17}$

In order to reach the upper part of the ureter with semi-rigid ureteroscopy, it is thought that general anesthesia will be more suitable as it relaxes the body. However, in this study, it was observed that the preference of general anesthesia for proximal ureter stones did not increase the success of semi-rigid ureteroscopy. Sen et al. also reported that the administration of general anesthesia had no effect on semi-rigid URSL success for proximal ureteral stones. ${ }^{2}$

There are many studies showing that using Ho: YAG laser for lithotripsy increases the success of semi-rigid URSL in proximal ureter stones. ${ }^{2,18,19}$ Over the time, lithotripter techniques have increased success in proximal ureter stones and decreased complication rates. In this study, Ho:YAG laser lithotripter was seen to be more effective than pneumatic lithotripter. Laparoscopic techniques are applied successfully for large stones for the upper ureter. ${ }^{17}$ The use of equipment, such as stone cone to prevent the push-back of the stone to proximal, increased the success of semi-rigid ureteroscopy.

Unlike other studies, the distance to UPJ and the ureter width in the proximity to the stone are evaluated in this study. The closer the stone is to the UPJ, the greater the failure. Therefore, when the stone is closer to the UPJ, it will be better to use stone cone and Ho: YAG laser to increase the success of the surgery. Moreover, if possible, flexible ureteroscopy should be used to reach stones that could be push-back. In this study, the proximal diameter of the ureter proximity to stone was found to be insignificant in terms of its effect on the success of the operation. In this study, D-J stent was placed on the majority of patients. Günlüsoy et al. preferred 
to put D-J stents on fewer patients; ${ }^{12}$ whereas, Şen et al. preferred to use D-J stents in more patients. ${ }^{2}$

The biggest limiting factor of this study was retrospective collection of the patient groups which are relatively small sized. The authors believe that prospective studies with a larger group of patients are needed to demonstrate the importance of semi-rigid URSL in the treatment of proximal ureter stones, and the factors affecting the success.

\section{CONCLUSION}

Semi-rigid URSL is a useful technique in the proximal ureter stones. It is useful to use Ho:YAG lithotripsy and stone cone to increase the success of semi-rigid URSL. The distance of the stone to the UPJ affects the success. Considering that failure may be more in stones closer to UPJ, it is useful to perform surgery in centres with flexible ureterorenoscopy.

\section{ETHICAL APPROVAL:}

The present study is approved by the Hacıbektaş University Clinical Research Ethical Committee. All procedures performed in this study, involving human participants, were in accordance with the ethical standards of the Institutional and National Research Committee and with the 1964 Helsinki Declaration and its later amendments or comparable ethical standards.

\section{PATIENTS' CONSENT:}

Since the study is conducted retrospectively, getting the patient consent in person was not applicable. Nonetheless, even before the Ethical Committee application, proper authorisations were taken from Nevşehir State Hospital administration by ensuring the patients' anonymity and protection of the patient data.

\section{CONFLICT OF INTEREST:}

The authors declared no conflict of interest.

\section{AUTHORS' CONTRIBUTION:}

SO: Collaborated in data processing, data analysis, and authored the manuscript.

SO, HK: Conceptualised and designed the study, edited and critically reviewed manuscript.

MCT, SO, NÖK: Initiated, conceptualised and designed the study, edited and critically reviewed manuscript.

CY, HK: Interpreted results and critically reviewed manuscript.

SO, MCT: Collaborated in collection of data and critically reviewed manuscript.

SO, HK, MCT, NÖK: Edited, critically reviewed manuscript and collaborated in quality control. SO: Performed statistical analysis and authored the manuscript.

All authors read and approved the final manuscript.

\section{REFERENCES}

1. Washino S, Hosohata K, Miyagawa T. Roles played by biomarkers of kidney injury in patients with upper urinary tract obstruction. Int J Mol Sci 2020; 21(15):5490. doi: 10.3390/ijms21155490.

2. Sen V, Irer B, Erbatu O, Yildiz A, Ongun S, Cinar O, et al. Predictive factors of ureterorenoscopy outcomes in proximal ureteral stones: A multicenter study of aegean study group of the society of urological surgery. Urol Int 2019; 104(1-2): 125-30.doi: 10.1159/000504790.

3. Türk C, Petrik A, Seitz C, Sarica K, Skolarikos A, Straub M, et al. EAU guidelines on urolithiasis. European Urol 2020; 69(3):475-82. doi: 10.1016/j.eururo.2015.07.041.

4. Wang $Y$, Chang $X$, Li J, Han Z. Efficacy and safety of various surgical treatments for proximal ureteral stone $>/=10 \mathrm{~mm}$ : A systematic review and network meta-analysis. Int Braz J Urol 2020; 46(6):902-26. doi: 10.1590/S1677-5538.IBJU.2019.0550.

5. Solakhan M, Seckiner SU, Seckiner I. A neural networkbased algorithm for predicting the spontaneous passage of ureteral stones. Urolithiasis 2020; 48(6):527-32. doi: 10.1007/s00240-019-01167-5.

6. Bangash M, Nazim SM, Jamil S, Abdul Ghani MO, Naeem S. Efficacy and safety of semi-rigid ureteroscopic lithotripsy (URS) for proximal ureteral stone $>/=10 \mathrm{~mm}$. J Coll Physicians Surg Pak 2020; 30(10):1058-62. doi: 10.29271/jcpsp.2020.10.1058.

7. Kartal I, Baylan B, Cakici MC, Sari S, Selmi V, Ozdemir H, et al. Comparison of semirigid ureteroscopy, flexible ureteroscopy, and shock wave lithotripsy for initial treatment of 11-20 mm proximal ureteral stones. Arch Ital Urol Androl 2020; 92(1):39-44. doi: 10.4081/aiua.2020.1.39.

8. Zheng J, Wang Y, Chen B, Wang H, Liu R, Duan B, et al. Risk factors for ureteroscopic lithotripsy: a case-control study and analysis of 385 cases of holmium laser ureterolithotripsy. Wideochir Inne Tech Maloinwazyjne 2020; 15(1):185-91. doi: 10.5114/wiitm.2019.85360.

9. Ogreden E, Demirelli E, Aksu M, Tok DS, Oguz U. Early ureteroscopic lithotripsy in acute renal colic caused by ureteral calculi. Int Urol Nephrol 2020; 52(1):15-9. doi: 10.1007/s11255-019-02298-9.

10. Sancak EB, Kilinc MF, Yucebas SC. Evaluation with decision trees of efficacy and safety of semirigid ureteroscopy in the treatment of proximal ureteral calculi. Urol Int 2017; 99(3):320-5. doi: 10.1159/000474954.

11. Wolf JS, Jr. Treatment selection and outcomes: Ureteral calculi. Urol Clin North Am 2007; 34(3):421-30. doi: 10.1016/j.ucl.2007.04.010.

12. Gunlusoy B, Degirmenci T, Kozacioglu Z, Arslan M, Ceylan Y, Nergiz N, et al. Factors affecting the complications of pneumatic lithotripsy for the treatment of ureteral stones with different localisations: A multivariate analysis of complications. Urol Int 2013; 91(3):357-62. doi: 10.1159/0003 50243.

13. Chen CS, Wu CF, Shee JJ, Lin WY. Holmium: YAG lasertripsy with semirigid ureterorenoscope for upper-ureteral stones $>2 \mathrm{~cm}$. J Endourol 2005; 19(7):780-4. doi: 10.1089/end. 2005.19.780.

14. Cheung MC, Lee F, Yip SK, Tam PC. Outpatient holmium laser lithotripsy using semirigid ureteroscope. Is the treatment outcome affected by stone load? Eur Urol 2001; 
39(6): 702-8. doi: 10.1159/000052530.

15. Seitz C, Tanovic E, Kikic Z, Fajkovic H. Impact of stone size, location, composition, impaction, and hydronephrosis on the efficacy of holmium:YAG-laser ureterolithotripsy. Eur Urol 2007; 52(6):1751-7. doi: 10.1016/j.eururo.2007.04.029.

16. Rana RS, Ather MH. Hounsfield units-a significant predictor of lasering time and energy in the management of upper urinary tract stones using Holmium: Yttrium-Aluminum Garnet lasers. Int Urol Nephrol 2020; 52(9):1637-41. doi: 10.1007/s11255-020-02442-w.

17. Kaygisiz O, Coskun B, Kilicarslan H, Kordan Y, Vuruskan H, Ozmerdiven $\mathrm{G}$, et al. Comparison of ureteroscopic laser lithotripsy with laparoscopic ureterolithotomy for large proximal and mid-ureter stones. Urol Int 2015; 94(2):205-9. doi: 10.1159/000368374.

18. Degirmenci T, Gunlusoy B, Kozacioglu Z, Arslan M, Koras O, Arslan B, et al. Comparison of Ho:YAG laser and pneumatic lithotripsy in the treatment of impacted ureteral stones: an analysis of risk factors. Kaohsiung J Med Sci 2014; 30(3):153-8. doi: 10.1016/j.kjms.2013.08.007.

19. Kassem A, Elfayoumy $H$, Elsaied W, Elgammal M, Bedair A. Laser and pneumatic lithotripsy in the endoscopic management of large ureteric stones: A comparative study. Urol Int 2012; 88(3):311-5. doi: 10.1159/000336254. 